\title{
Primes and flankers: Source confusions and discounting
}

\author{
Stephen E. Denton • Richard M. Shiffrin
}

Published online: 14 April 2012

(C) Psychonomic Society, Inc. 2012

\begin{abstract}
Visual identification of briefly presented target words is affected by the presence of nondiagnostic prime words that immediately precede the target, flanker words simultaneously presented adjacent to the target, and visual masks that immediately follow the target in the same location. Priming is duration dependent: In a forced choice target identification task, brief primes produce a strong preference to choose the primed alternative, whereas long primes have the opposite effect. The ROUSE model (Huber, Shiffrin, Lyle, \& Ruys, Psychological Review 108:149-182, 2001) predicts this interaction by positing that prime features are confused with target features and that evidence regarding the prime features is discounted less for short primes and more for long primes, when both are compared with the optimal level. In the present study, we augmented the typical short-term priming experiment by adding flankers that appeared simultaneously with the target and remained for a short or long duration. In the experiment, we replicated previous priming effects and produced novel effects of flanker duration. ROUSE accounted for both the priming and flanker findings with the previously posited processes, but with different quantitative parameters for flankers: Relative to optimal levels of discounting, all flanker features were underdiscounted, but longer flankers were discounted more than short flankers.
\end{abstract}

Keywords Word perception $\cdot$ Priming $\cdot$ Math modeling

\footnotetext{
S. E. Denton $(\bowtie)$

Department of Psychology, Vanderbilt University, Nashville, TN, USA

e-mail: sedenton@umail.iu.edu

R. M. Shiffrin

Department of Psychological and Brain Sciences, Indiana University,

Bloomington, IN, USA
}

In the present article, we attempted to bridge two common paradigms used to study visual perception-priming and flanking — and to tie both to a third, masking. These threads are drawn together with a theory that assumes feature migration and confusions among stimuli close together in time and space, and a discounting mechanism that attempts to offset these confusions by assigning differential levels of evidence to features that are in primes and flankers. The theory is an extension of the ROUSE (Responding Optimally with Unknown Sources of Evidence) theory (Huber, Shiffrin, Lyle 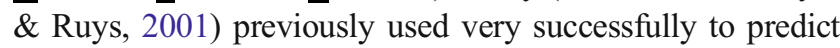 visual priming.

Short-term priming refers to a well-known finding that a prime stimulus presented just prior to a target stimulus influences its perception, even when it is nondiagnostic for the target detection task. A particularly important finding is the fact that the direction of priming is changed by the duration of a prime and the attention given to it. Short and less attended primes (even "subliminal" ones) produce positive priming - a tendency to think the target matches or is similar to the prime. Long and more attended primes produce negative priming - a tendency to think the target is not the prime and not similar to it. Such effects are seen in both accuracy of detection (say, in naming, yes-no matching, or forced choice, e.g., Hochhaus \& Johnston, 1996) and response time (RT). In a long series of studies, Huber, Shiffrin, and colleagues surveyed much of the research on priming, carried out much additional research of their own, and produced a very effective model, termed ROUSE, that explains the results (Denton \& Shiffrin, in press; Huber, 2008; Huber, Shiffrin, Lyle \& Quach 2002a; Huber et al., 2001; Huber et al., 2002b; Weidemann, Huber \& Shiffrin, 2005, 2008). To keep the present article short, we refer the readers to those articles for much of the background and details concerning priming and its modeling. 
The essence of the ROUSE model is rooted in two factors. First, features of primes are posited to migrate into the percept of the subsequent target stimulus, without (full) knowledge of the source of such features. Thus, the system/ observer cannot be sure that the target percept includes features only of the target itself; some of those features could have come from the prime. The degree to which this migration occurs presumably depends on the prime's salience, which is a function of its duration and the attention given to it. Second, the evidence assigned to a percept feature takes into account the possibility that the feature was from a prime rather than a target. The evidence provided by percept features depends on the extent to which the system "knows" that the prime could have been a source. The features of a long and attended prime are salient and therefore may have a high tendency to join the target percept, but such migrations are obvious to the system. Consequently, the evidence for those features, when found in the target percept, can be "discounted" strongly on the basis that they could have arisen from the prime and not the target. On the other hand, the features of a short, unattended, or even "subliminal" prime may contribute few features to the target percept, but the source of those features are less obvious to the system; therefore, the evidence for those features are less strongly discounted. If the probability that a feature from a prime joins the target percept were discounted to exactly the right degree, then primes would produce no tendency toward positive or negative priming. However, ROUSE predicts the observed findings by assuming that short, less attended primes are underdiscounted, leading to positive priming, and that long, more attended primes are overdiscounted, leading to negative priming.

The ROUSE model does an excellent job of accounting for a variety of priming findings, but one might well ask whether the same mechanisms come into play when nondiagnostic stimuli occur not prior to the target but simultaneously with it, or even after it. Such paradigms have a long history of study in the field and are usually termed flanker studies (e.g., Eriksen \& Eriksen, 1974). In the standard flanking experiment, a stationary target is presented in a predefined location. The target stimulus appears with other stimuli that flank it on one or more sides. The flanker stimuli can be congruent with target stimuli, either by being perceptually similar to the target or associated with the same response option, or be incongruent by being associated with a different response than the target. The typical flanker effect is that target identification is slower and less accurate when the flanker is incongruent with the target than when it is congruent (known as the flanker congruency effect). The presence of incongruent flankers interferes with the accurate and quick processing of a target item. In these studies, the congruency of the response mapping, or response compatibility, is thought to be a critical factor (see Eriksen, 1995).
Explanations of flanker effects often postulate that the flanker causes automatic activation of the response channel associated with the flanker stimuli (e.g., Gratton, Coles \& Donchin, 1992; Gratton, Coles, Sirevaag, Eriksen \& Donchin, 1988). In incongruent conditions, this automatic activation leads to fast incorrect responding or must be overruled by some process that ultimately slows correct responding. The features of the flankers need to be processed and mapped to responses to produce congruency effects. Much evidence supports the hypothesis that response compatibility plays an important role in flanking effects, but other factors might also play a role. In particular, the degree to which flanking stimuli produce features that join the target percept, and then become discounted, is unknown. Flanker features that are compatible with a particular response might migrate to the target percept and contribute to congruency effects without directly activating response channels.

Research on priming and flanking has tended to occupy two different scientific niches, but the paradigms are in many ways remarkably similar, and both can be seen as studies of the interaction of targets and other stimuli that are nearby in time and space. Even when primes and flankers are nondiagnostic and, in that sense, irrelevant to the target identification task, the visual system cannot help registering features of the prime and flanker stimuli because of their spatial and temporal proximity; to some degree, this occurs automatically.

In the present study, we therefore attempted to gain insight into the processes that might be common to priming and flanking effects by including both procedures in a common task on the same observers. Furthermore, we hoped to account for effects of both with a common model framework, one building upon the prior successes of the ROUSE model. The idea was to view flankers as yet another source of features that might join the target percept. There is every reason to believe that primes and flankers will differ both in the degree to which their features migrate to the target percept and in the degree of discounting. Nonetheless, the processes in ROUSE should apply to flankers, even though the values of the migration and discounting parameters should differ from those for primes.

It is important to note our use of terminology. In previous studies, flankers are spatially adjacent and contemporaneous; primes are temporally prior, but have spatial positions relative to the target that vary. In the rest of the present article, we used flankers to refer to the nontarget stimuli that begin simultaneously with the target, and primes to refer to nontarget stimuli that begin (and end) prior to the target.

In the present experiment, we used both prime and flanking stimuli in combination with a central flashed target that was then postmasked and followed by two choices. The target was always included in the choices with another foil 
choice. Both the target and the foil choice could variously match primes, flankers, neither, or both. The measure of interest was choice accuracy, not RT. This design should have minimized any effects resulting from response compatibility. Although the possibility of such effects could not be entirely be ruled out, we considered only models for the present studies in which the effect of primes and flankers was the result of feature migration into the target percept, and discounting evidence derived from those features.

\section{Short-term priming paradigm}

The tasks used in the present article were based on those used initially by Huber et al. (2001) to study priming. Two prime words of variable duration were followed by a flashed and postmasked target word so that target perception was near threshold. The target flash was followed by a twoalternative forced choice between the target and a foil. In three critical conditions, the primes were identical and could match the target only (target-primed condition), the foil only (foil-primed condition), or neither choice (neither-primed condition). These conditions were mixed so that the primes were nondiagnostic on average and therefore irrelevant to performance; the prime was just as likely to indicate the incorrect choice as the correct choice. Participants were informed that the primes were irrelevant, and the trial-bytrial feedback demonstrated this to be the case.

Huber, Shiffrin, and colleagues (Huber, Shiffrin, Lyle \& Quach, 2002; Huber et al., 2001; Huber et al., 2002a; Huber et al., 2002b; Weidemann et al., 2005, 2008) demonstrated the ease with which priming effects can be manipulatedfor example, using prime duration to change their magnitude and direction. When primes appeared for a short duration, there was a positive priming preference - a tendency to select the choice that had been primed regardless of whether it was correct (as in the target-primed condition) or incorrect (as in the foil-primed condition). When the primes appeared for longer duration, this preference reversed: There was a tendency to select whatever choice had not been primed. The ROUSE model that was proposed by Huber et al. (2001), and that was substantiated in a variety of subsequent studies, accounts for these duration-dependent priming effects with two offsetting mechanisms: (a) Features migrate from the prime to the target percept, without source identification, producing source confusion, and (b) evidence for features known to have been in primes is discounted, to a degree that is slightly too high for long primes and slightly too low for short primes, both relative to the optimal level.

Although these mechanisms have much explanatory power, it remains unclear whether factors that control feature migration are the same as those that control discounting. In previous work, researchers have explored the nature of sequential prime presentation with multiple primes all appearing before the target flash. Weidemann et al. (2005) found that two primes presented sequentially (long, then short) at different spatial locations acted as independent feature sources. Features from both primes migrated into the percept and additively combined to produce source confusion, but features from the long prime were discounted to a much greater extent. These results show that spatial and temporal factors are important for both feature migration and discounting, and suggest that features from sources at different spatial and temporal locations will have independent effects. However, these results do not directly speak to the question of what the resulting effects will be for feature sources that are cotemporal with target features.

\section{Source confusion and discounting of flanker features}

Supposing that flanking stimuli are added to the priming task described above, it seems reasonable to assume that these will produce features that will migrate into the percept, and that such features will be discounted. However, the degree to which each of these factors will operate will almost certainly differ from those for primes. To take an extreme example, if flankers were made to onset at times successively later that the target flash, the migration of features to the target percept would certainly drop to an increasing degree and the need for, and the actual presence of, discounting would similarly drop. In fact, the importance of order in feature migration might cause the system to lower the degree of discounting of flanking features, even beyond that required by the actual feature migration. If so, both short and long flankers might be discounted less than optimally, although one would still expect more discounting for longer flankers.

\section{Combining primes and flankers}

In the present experiment, we not only studied flanking effects in a two-alternative forced choice target detection paradigm, we did so in a study that included both priming and flanking stimuli on each trial. As usual, neither flankers nor primes were diagnostic for the correct choice, but the relation between flankers, primes, their durations, and the two choices was co-varied. The cost of this design is a complex study with a very large number of conditions, but the benefits include a way to compare directly effects of flankers and primes, and data that should highly constrain possible models (including both the variants of ROUSE that have been published and also alternatives to ROUSE that have yet to be formulated). 
Note that flanking as was used in this paradigm departs from typical flanking paradigms in several respects. The target was a common word that was not disclosed before the beginning of the trial. This design differs from the standard flanking experiment in which targets are known to be from a limited set (generally letters or arrows), and the mapping from particular set items to response options is given at the beginning of the experiment. Also, at the time flanker words were presented in the present paradigm, the flankers were not yet associated with particular responses. Similar to the standard priming conditions, we included three flanking conditions. The flanker could be identical to the target (target-flanked condition), the flanker can be identical to the foil (foil-flanked condition), or it can be unrelated to either (neither-flanked condition). The targetflanked condition was roughly equivalent to a congruent flanker, because the features from the flanker were compatible with the correct response. The foil-flanked condition was similar to an incongruent condition, because the features activated by the flanker provided evidence for the incorrect response. Since the duration of the prime determines the nature of the priming effect, we included two flanker duration conditions to investigate any flanker duration-dependent effects that were possibly the results of differential discounting. These flanking conditions were covaried with similar priming conditions.

\section{Masking}

The paradigm we used employed masks directly, in the form of pattern masks that immediately followed the target flash in the same location, referred to as backward form masking. Previous research in the present priming paradigm and similar ones (Sanborn, Malmberg \& Shiffrin, 2004) have demonstrated that such masks add noise to the lower level form features extracted from the target flash, so that the decision between the two choices is based on higher level features such as the more abstract "names" of the features and stimuli presented. For example, a target presented in a particular case (upper or lower) does not improve performance when the target choice is in the same case and the alternative choice in different case. The same result obtains when color rather than case is manipulated and the mask has multiple colors. Thus, in the models discussed and presented, features generally will refer (implicitly) to such higher level and more abstract features. Other research has similarly noted that priming effects are attributable to prelexical, yet not perceptual, orthographic representations encoding letter identities (Kinoshita \& Norris, 2009; Norris \& Kinoshita, 2008)

It should be noted that masking appears indirectly in our paradigm, in the form of forward masking (the primes can be thought of in this fashion) and backward/metacontrast masking (the flankers can be thought of in this fashion). The studies were not aimed at masking per se, and masking was not varied across the conditions except for the manipulations of primes, flankers, and their durations, but the literature on masking does provide insight into the findings. Such discussion will be deferred until the studies, results, and models have been presented.

\section{Summary}

The empirical focus of the present experiment was an investigation of the degree to which flankers are sources of features that join the target percept, and, if so, the degree to which the evidence assigned to such features are discounted. In the study, we assessed the effect of duration of both primes and flankers. By using both primes and flankers in the same study, we could compare the results quantitatively and make inferences about the comparative strength of feature migration and discounting in the two cases. Theoretically, we wished to assess whether the processes of ROUSE allowed prediction of the combined results.

\section{Priming and flanking experiment}

The sequence of events for two example trials is depicted in Fig. 1. First, the prime word was presented in duplicate in the center of the screen for either $50 \mathrm{~ms}$ or $1,000 \mathrm{~ms}$. The prime word could be either the same as the target, the foil, or neither, corresponding to the prime conditions discussed earlier. The target word was then flashed at the center of the screen. A flanker word appeared both above and below the target. Similar to standard priming conditions, the flanker word could be the same word as the target, foil, or neither, corresponding to the three flanker conditions: target flanked, foil flanked, and neither flanked.

Since the flanker and target were the same word in the target-flanked condition, we had some concern that these trials would be qualitatively different from other conditions, because perceptually the target word would appear in triplicate. To investigate any possible effects of superficial lowlevel letter similarity effects, we included a flanker lettercase manipulation. On half of the trials, the flanker word appeared in all uppercase letters, which is how the target, prime, and foil were presented. On the other half, the flanker word appeared in all lowercase letters. The lowercase flankers were no longer superficially identical to the target (or foil), but would presumably still activate the same higher level letter and word features. Following the presentation of the target flash, flankers, and mask, the target and a foil were 


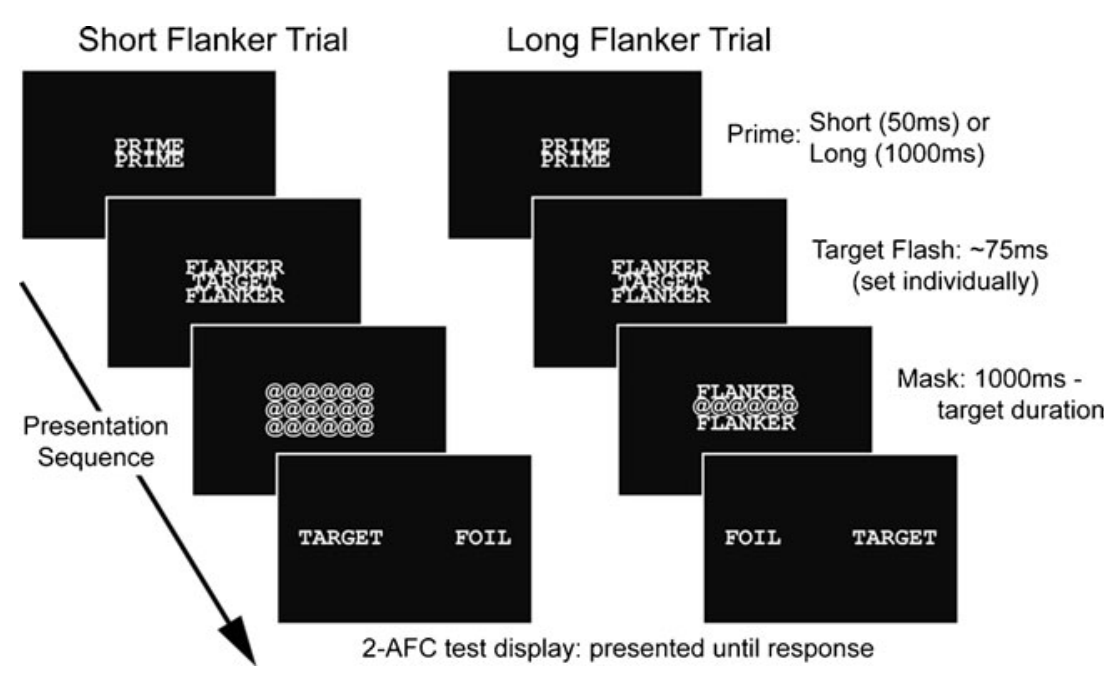

Fig. 1 Two example trial sequences are presented. The left sequence shows a short flanker trial, and the right shows a long flanker trial. Frame durations are presented on the right. The prime could be either short or long in both sequences. The only difference between the two sequences was that the flankers in the long flanker condition stayed present when the target was masked and were not themselves masked (the choice screen appeared at their offset). All sequences were preceded by a $500-\mathrm{ms}$ presentation of a fixation cross in the center of the screen (not shown). The positions of the target and foil word on the last frame were randomized presented. The participant's task was to select the target. An important component of the present study, as in previous priming studies, was the effect of duration. Would short presentations of the flanker produce a different pattern of effects than long? Would the results be interpretable as being due to a difference in the probability that flanker features migrate into the target percept or a difference in the estimate of that probability used when discounting evidence? To investigate this possibility, two flanker duration conditions were included. The flanker word that appeared both above and below the target could appear for a short duration, the same duration as the target flash, or it could be presented for a long duration, $1 \mathrm{~s}$. Figure 1 depicts one trial for both the short and long flanker conditions; a schematic of a short flanker trial appears on the left and a long flanker trial appears on the right.

In the present experiment, six factors were crossed within subjects. These included the five factors already discussed: priming condition (three levels: target, foil, or neither primed), flanker condition (three levels: target, foil, or neither flanked), prime duration (two levels: short or long), flanker duration (two levels: short or long), and flanker letter case (two levels: upper- and lowercase). When crossed, these five factors produced 72 different types of trials, which were each presented once per block in a random order. Additionally, two word lengths were used: either five or six letters long. This word-length factor was varied across blocks, so that participants would alternate between fiveletter word blocks (of 72 trials) and six-letter word blocks. Within a block, all words (primes, flankers, targets, and foils) were the same length.

\section{Method}

Participants A total of 104 undergraduate students volunteered to participate for partial credit in an introductory psychology course at Indiana University.

Materials and apparatus We used two pools of 1,100 fiveletter and 1,600 six-letter words with a written-language frequency of at least three per million as defined by Kučera and Francis (1967). All words were presented using the fixed-width Courier Bold 17-point font. The primes, targets, foils, and half of the flankers appeared in uppercase, whereas the other half of the flankers appeared in lowercase. All of the masking was done with rows of six "@” signs presented in Arial Narrow Bold 13-point font. This ensured dense coverage of the prime, target, and, in the appropriate conditions, flankers. All of the stimulus words and masks were presented in white against a black background.

Stimulus words were sorted into sets of two, three, or four words separately for each participant and each word length (five or six letters). This sorting was done to minimize letter overlap between words presented on the same trial. On neither-primed, neither-flanked (NP/NF) trials, four separate words were needed as the prime, target, flanker, and foil. It was not possible to eliminate letter repetitions across these four words, but within each word set, at most one letter overlapped across two words. This one letter was not in the same position within the two words, and all other letters were unique. For each participant and word length, 32 sets of four words, 128 sets of three words, and 128 sets of two words were generated by selectively choosing words 
from a list (either a five- or six-letter word list). Based on the combination of prime and flanker types for a particular trial, words were sampled from the appropriate list without replacement so that each word appeared at most once for each participant, and the possibility of long-term repetition priming was eliminated.

All of the stimuli were displayed on 16-in. $(40.6 \mathrm{~cm}) \mathrm{PC}$ CRT monitors with vertical refresh rates of $120 \mathrm{~Hz}$ and a screen resolution of $800 \times 600$ pixels. The experiment was programmed using the Vision Egg library for the python programming language (Straw, 2008). The display was synchronized to the vertical refresh of the monitor providing display increments of $8.33 \mathrm{~ms}$. Participant responses were collected using a standard computer keyboard.

Procedure Participants sat individually in a dimly lit, ventilated, sound-dampened booth. Each trial began with a fixation cross appearing in the center of the screen for $500 \mathrm{~ms}$. The prime was then presented in the location indicated by the fixation cross. The prime was always presented as the same word displayed twice, one directly atop the other, following the standard procedure used in prior research (see Fig. 1). The prime was either short $(50 \mathrm{~ms})$ or long $(1,000 \mathrm{~ms})$. The target word appeared immediately after the prime offset and was displayed for a short period (individually adjusted, but approximately $70 \mathrm{~ms}$ for the average participant; see below). The target was then masked. Although the duration of the target flash varied between participants, the duration of the target and the mask together equaled $1,000 \mathrm{~ms}$ (i.e., mask duration $=1,000 \mathrm{~ms}-$ target duration). The onset of the flanker words was simultaneous with the onset of the target. In the short flanker condition, the offset of the flankers was simultaneous with the offset of the target. In the long flanker condition, the flankers remained after the target was masked. The long flankers appeared for exactly $1,000 \mathrm{~ms}$ and then were removed (but not masked: Long flankers were clearly visible and ended well after the target flash, so masking should not have been relevant). After the target mask offset, two choice words appeared to the right and left of the target location. One choice was the target, whereas the other was a foil. The location of the target choice was randomized across trials, appearing equally often on the left and right. Participants were asked to identify the target word in this two alternative forced choice (two AFC) test by pressing the "F" or " $\mathrm{J}$ " key to choose the left or right alternative, respectively. Once a selection was made, feedback was provided.

Each participant went through 624 priming trials broken into nine blocks. The first block was made up of 48 trials, whereas the remaining eight consisted of 72 trials each. The first 16 trials were NP/NF practice trials with long target durations $(150 \mathrm{~ms})$ to get participants used to the task. These practice trials were followed by $32 \mathrm{NP} / \mathrm{NF}$ calibration trials.
Target word durations were individually adjusted for each participant so that accuracy was roughly $75 \%$ in NP/NF conditions. This calibration was done using a staircase method. As with previous studies (e.g., Huber et al., 2001; Weidemann et al., 2005), there were large individual differences. Target flash times ranged from $25 \mathrm{~ms}$ to $200 \mathrm{~ms}$ (the maximum allowed), with a median of $66.7 \mathrm{~ms}$. The practice and calibration trials in Block 1 were not analyzed further.

\section{Results}

The data of interest were the accuracy measures, but RTs were used to help eliminate suspect participants and trials. Any participant whose overall performance was not significantly above chance, given an exact binomial test - which in the present context yields a cut-off of $54.2 \%$ correctwas eliminated. Extreme RTs were defined to be those less than $100 \mathrm{~ms}$ or more than $5 \mathrm{~s}$. Participants with extreme RTs on more than $5 \%$ of trials were eliminated. These criteria (accuracy and extreme response time) together eliminated 13 out of the 104 participants, leaving 91 for further analysis. For the remaining participants, trials with extreme RTs were eliminated from further analysis (roughly $0.4 \%$ of these participants' data).

A 3 (priming condition) $\times 3$ (flanker condition) $\times 2$ (prime duration) $\times 2$ (flanker duration) $\times 2$ (flanker case) $\times$ 2 (word length) six-way within-subjects ANOVA was performed on the accuracy data from all remaining participants. Two word lengths were included in the present study only because of the limited number of relatively common words available that were five or six letters in length. It was not expected that one letter would produce any difference in participant performance and, as was expected, there was no main effect of word length, and this factor did not interact significantly with any other factor. As such, this factor was removed from the analysis.

The significant effects of the remaining five factors are presented in Table 1. As can be seen in the table, and as was expected on the basis of prior research, there were many significant effects. The main effect of flanker case (either upper or lower) was significant. This main effect was due to performance being generally worse on trials in which the flanker was presented in lowercase. This effect was the greatest when target was flanked by the foil word; that is, the decrease in lowercase performance was worse on foilflanked conditions than on either target- or neither-flanked conditions. Thus, flanker case had a significant interaction with flanker condition. However, no other interactions between flanker case and other factors were observed. In the remainder of the paper, we ignore the effect of flanker case, as it is not deemed particularly important for present purposes, and average across upper- and lowercase flanker words. 
Table 1 Statistical test results

\begin{tabular}{|c|c|c|c|}
\hline Effect & $\left(d f_{N}, d f_{D}\right)$ & $M S E$ & $F$ \\
\hline Priming Condition & $(2,180)$ & 0.074 & $34.2 * *$ \\
\hline Flanker Condition & $(2,180)$ & 0.079 & $166^{* *}$ \\
\hline Prime Duration & $(1,90)$ & 0.083 & $139 * *$ \\
\hline Flanker Duration & $(1,90)$ & 0.040 & $236^{* *}$ \\
\hline Flanker Case & $(1,90)$ & 0.024 & $36.2 * *$ \\
\hline $\begin{array}{l}\text { Priming Cond. } \times \text { Flanker } \\
\text { Con. }\end{array}$ & $(4,360)$ & 0.029 & $89.3 * *$ \\
\hline $\begin{array}{l}\text { Priming Cond. } \times \text { Prime } \\
\text { Dur. }\end{array}$ & $(2,180)$ & 0.092 & $285^{* *}$ \\
\hline $\begin{array}{l}\text { Flanker Cond. } \times \text { Prime } \\
\text { Dur. }\end{array}$ & $(2,180)$ & 0.029 & $9.70 * *$ \\
\hline $\begin{array}{l}\text { Priming Cond. } \times \text { Flanker } \\
\text { Dur. }\end{array}$ & $(2,180)$ & 0.033 & $57.3^{* *}$ \\
\hline $\begin{array}{l}\text { Flanker Cond. } \times \text { Flanker } \\
\text { Dur. }\end{array}$ & $(2,180)$ & 0.043 & $28.7 * *$ \\
\hline Flanker Cond. $\times$ Flanker Case & $(2,180)$ & 0.020 & $16.8^{* *}$ \\
\hline $\begin{array}{l}\text { Priming Cond. } \times \text { Flanker Cond. } \times \\
\text { Prime Dur. }\end{array}$ & $(4,360)$ & 0.028 & $19.8^{* *}$ \\
\hline $\begin{array}{l}\text { Priming Cond. } \times \text { Flanker Cond. } \times \\
\text { Flanker Dur. }\end{array}$ & $(4,360)$ & 0.020 & $3.38^{*}$ \\
\hline $\begin{array}{l}\text { Priming Cond. } \times \text { Prime Dur. } \times \\
\text { Flanker Dur. }\end{array}$ & $(2,180)$ & 0.036 & $21.6^{* *}$ \\
\hline $\begin{array}{l}\text { Flanker Cond. } \times \text { Prime Dur. } \times \\
\text { Flanker Dur. }\end{array}$ & $(2,180)$ & 0.027 & $26.6^{* *}$ \\
\hline
\end{tabular}

$* p<.01, * * \mathrm{p}<.001$

Although the remaining effects shown in Table 1 appear to be robust and interpretable, we will not go through the elaborate process of listing them here in the text. We believe it is most informative to present the results in graphical form and to use a model (an augmented version of the ROUSE model) to structure our interpretation of the results.

Study results are depicted in Fig. 2. This figure shows the mean performance across 91 participants in each of 36 within-subjects conditions. Each of the four panels shows a different combination of prime and flanker duration conditions (short-short, long-short, short-long, and longlong). Within each panel, all possible combinations of the three priming and three flanking conditions are presented. For example, the first bar in each panel shows the mean performance on trials in which the prime and flanker did not match either the target or foil (NP and NF). With 36 conditions, the trends in Fig. 2 can be difficult to interpret. Therefore, we show the marginal effect of the prime and flanker in Fig. 3. Each bar in Fig. 3 combines three bars from Fig. 2 by collapsing across either flanking or priming conditions.

The top row of Fig. 3 shows that we found the typical priming effects. When the prime duration is short, we find positive priming. Priming with the target improves performance relative to a neutral prime (the NP condition), whereas priming with the foil decreases performance. However when the prime is long, we see a reversal to negative priming. Priming with the target now reduces performance. Interestingly, the effect of the prime interacts with the duration of the flanker, so that positive prime appears the most pronounced with a short prime and a long flanker.

The bottom row of Fig. 3 isolates the effect of flanking condition across the four duration conditions. The main effect was positive, and the flanking effect never reverses and becomes negative. Nonetheless, duration had an effect because the positive flanking effect was reduced in the longflanker, short-prime conditions. This positive flanker effect is in the same direction as the flanker congruency effect (although the present research does not make it clear whether the mechanisms involved are the same). That is, flankers associated with the correct response (the target) lead to better performance than flankers associated with the incorrect response (the foil).

Figures 2 and 3 together provide a complex set of results. The prime and flanker conditions interact with prime and flanker duration in a number of interesting ways, as is also demonstrated by the many significant interactions in the data presented in Table 1. Understanding these complex trends is aided greatly by the model presented next, a model that explicates the processes producing the results.

\section{An augmented ROUSE model}

The ROUSE model was developed to account for a widerange priming effects. For detailed background and an exposition of the ROUSE model, the reader is referred to the prior articles cited in the introduction (particularly Huber et al., 2001, which gives the original version, and Huber, 2006, which gives a later analytic version). ROUSE assumes that the percept of the target flash contains not only features from the flash itself, but also features from the prime and the mask and other sources of noise. Furthermore, ROUSE assumes that the source from which these percept features arise is not known (this process referred to as source confusion). ROUSE then assesses evidence concerning the match of these percept features to choice options by discounting evidence from features based on the source that could have produced them. For example, if a prime was long and visible, and a feature in the percept was known to be in that prime, the evidence for a choice containing that feature would be discounted. More specifically, there exist probabilities that each of several possible sources contributed the features that appear in the percept. The system estimates those probabilities (but not perfectly) and discounts on the basis of those estimates. Positive priming is predicted on the basis of too low an estimate of the probability that a prime produces a percept feature, which occurs for short primes. Negative priming is predicted on the basis of too high an 
Fig. 2 Forced choice

performance and corresponding ROUSE predictions

(represented by the dots and crosses). The bar heights show the mean proportions-correct target identification choices (error bars show 95\% confidence intervals) within each condition. The four panels correspond to the four combinations of prime and flanker duration conditions: short primes on the left, long primes on the right, short flankers on the top, and long flankers on the bottom. Each panel is subdivided by flanker condition (along the $x$-axis) and priming condition (bar color). The dashed horizontal line indicates $75 \%$ performance: the accuracy that participants should roughly achieve on neither-primed/neither-flanked trials due to the target duration calibration procedure. The black dots $(\bullet)$ show the predictions of the full ROUSEa model. The crosses $(\times)$ show the predictions of a restricted ROUSEa model (see the text for details)
A1: Short Prime, Short Flanker

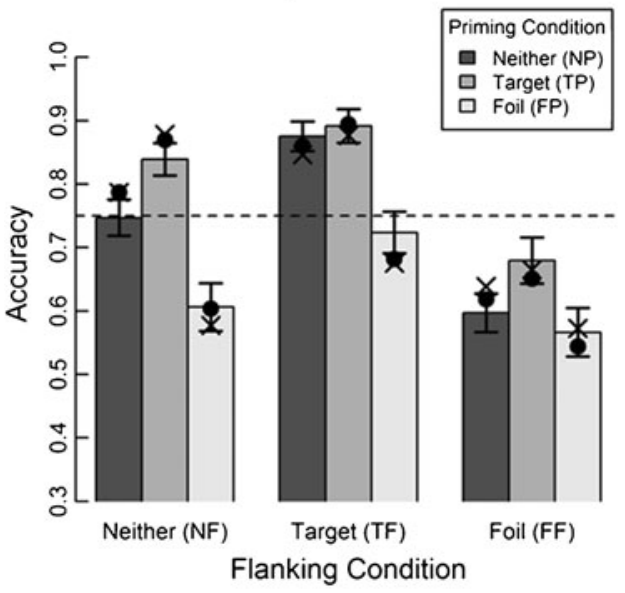

B1: Short Prime, Long Flanker

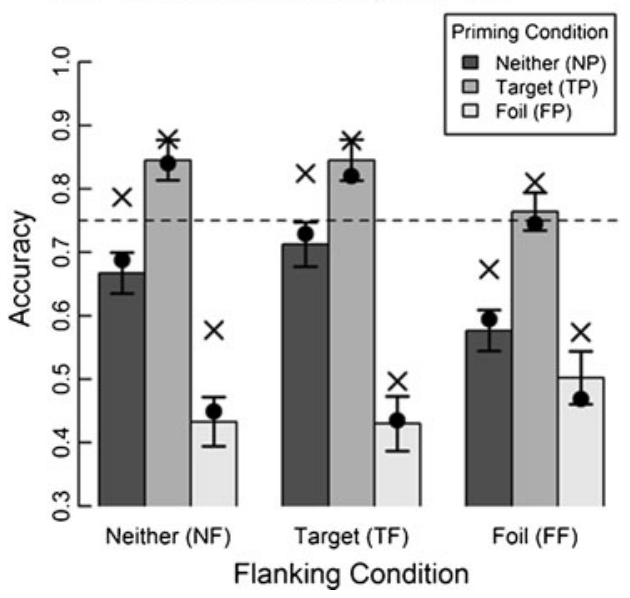

A2: Long Prime, Short Flanker

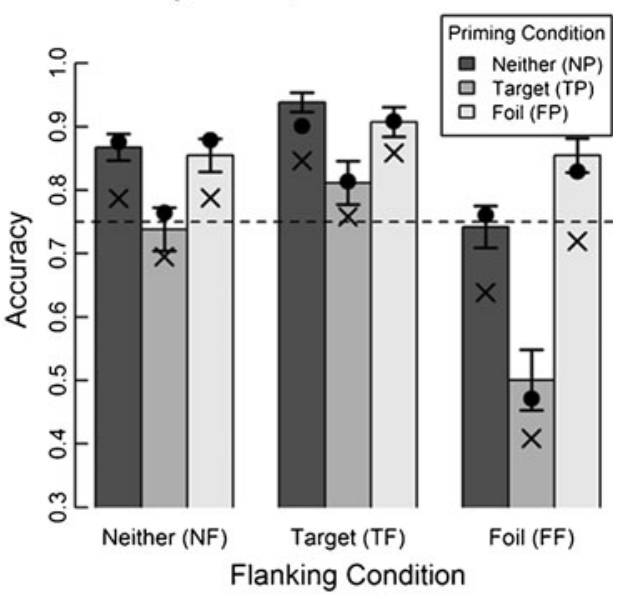

B2: Long Prime, Long Flanker

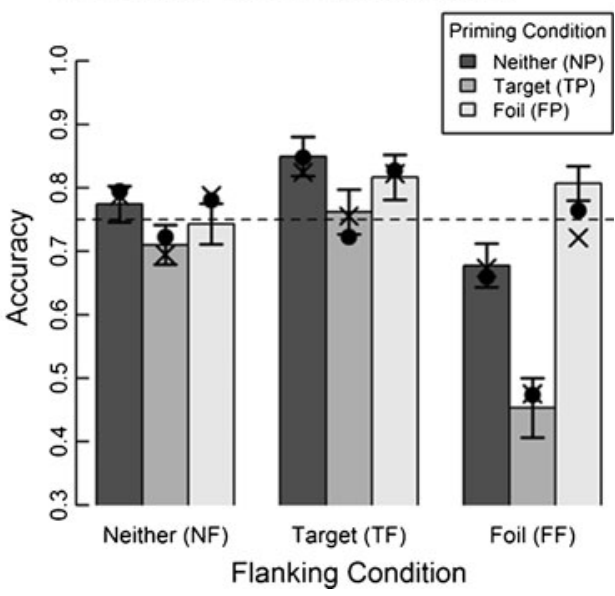

estimate of the probability that a prime produces a percept feature, which occurs for long primes. These assumptions and processes are left completely intact in the augmented version of ROUSE presented in this article.

The augmentation is quite simple and straightforward: Flankers are added as another source contributing features to the percept. The original ROUSE model assumes that the prime, target, and noise were all independent sources of features for the target percept. Because flankers in the experiment are spatially and temporally distinct from other feature sources, the augmented ROUSE model, termed ROUSEa in what follows, merely adds flankers to the list of independent feature sources that could enter into the target percept. The evidence provided by these additional intruding flanker features is evaluated in a manner similar to that of primes. That is, the model assumes a probability that a flanker contributes a feature and uses an estimate of that probability to discount evidence. As usual, these parameters depend on flanker duration. The addition of an independent feature source (flankers in the present case) does necessitate the addition of parameters to the model (with the accompanied increase in model complexity), but the extension is principled because the added flanking-related parameters are exactly analogous to the prime related parameters. Furthermore, all parameters are psychologically meaningful relating directly to intrusion rates and discounting rates for possible feature sources, to which flankers have naturally been appended. ROUSEa is depicted in Fig. 4. The figure shows the sources of features that join the flash percept and the parameters governing their probabilities. The prime and flanker probabilities are allowed to vary with prime and flanker duration, and the system uses estimates of the probabilities to discount evidence when the percept is matched to the two choices.

To be more explicit and exact, choice words (and percept) are represented as feature vectors. The features are thought to be somewhat abstract orthographic "names" because of the use of post masks (as was described in the original ROUSE papers and in the introduction of the present article; see Sanborn et al., 2004), although this assumption is not used explicitly in any of the derivations. ROUSEa represents words as feature vectors consisting of present or absent features; the length of these vectors is arbitrarily set to 20 (as in previous research). The features of the target and foil 
Fig. 3 Marginal forced choice performance obtained by collapsing across conditions in Fig. 2. Bar heights show mean proportion correct within conditions, and error bars show 95\% confidence intervals. Top row: Marginal priming effects obtained by collapsing across flanking conditions; the priming condition is indicated by the bar color. Bottom row: Marginal flanker effects obtained by collapsing across priming conditions; the flanking condition is indicated by bar color. For both rows, flanker duration conditions are presented in separate panels, and prime duration conditions appear on the $x$-axis. ROUSE predictions (shown as dots and crosses) were obtained by simply averaging over the appropriate conditions from Fig. 2

\section{A: Marginal Priming Effects}
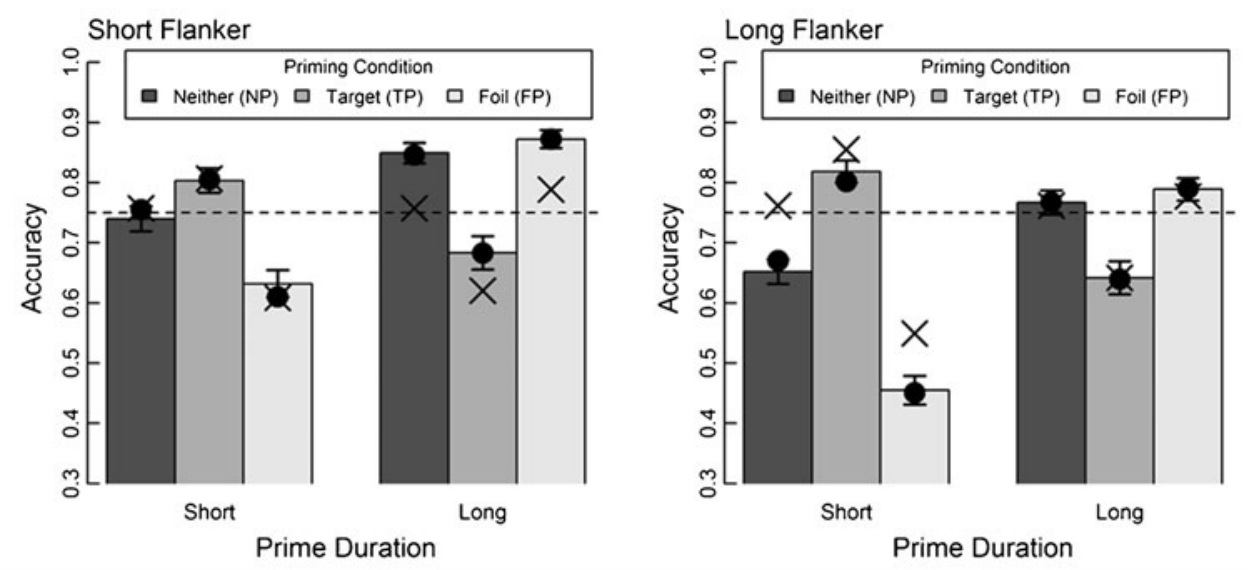

\section{B: Marginal Flanker Effects}

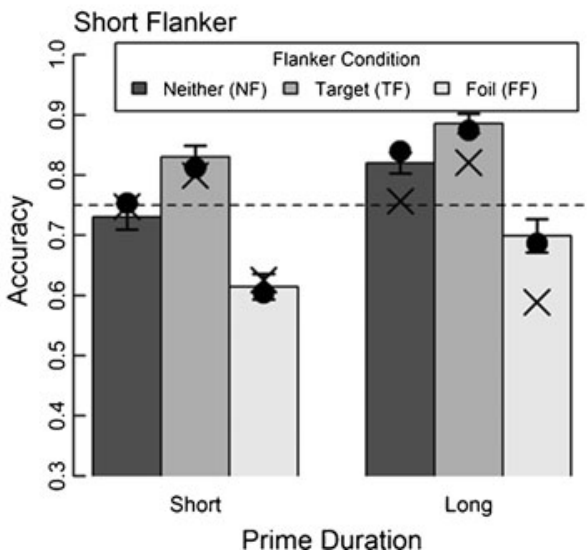

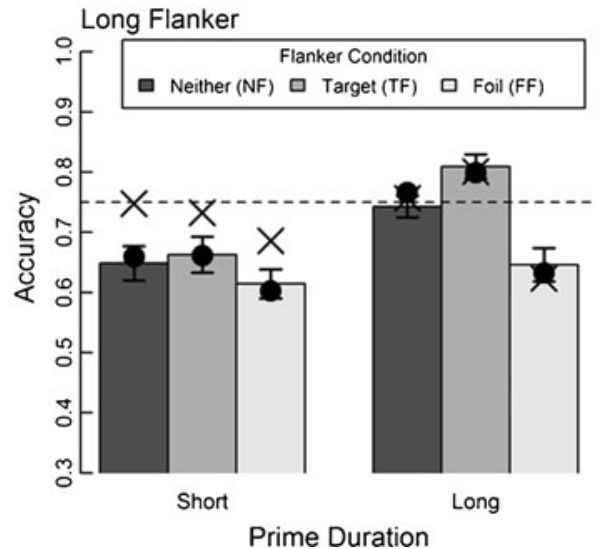

choices are matched against the current percept, with matching features providing evidence for that choice alternative and mismatching features providing evidence against that choice alternative. The percept is the result of stochastic feature activation from presentation of the prime, with probability $\alpha$; the flanker, with probability $\varphi$; the target, with

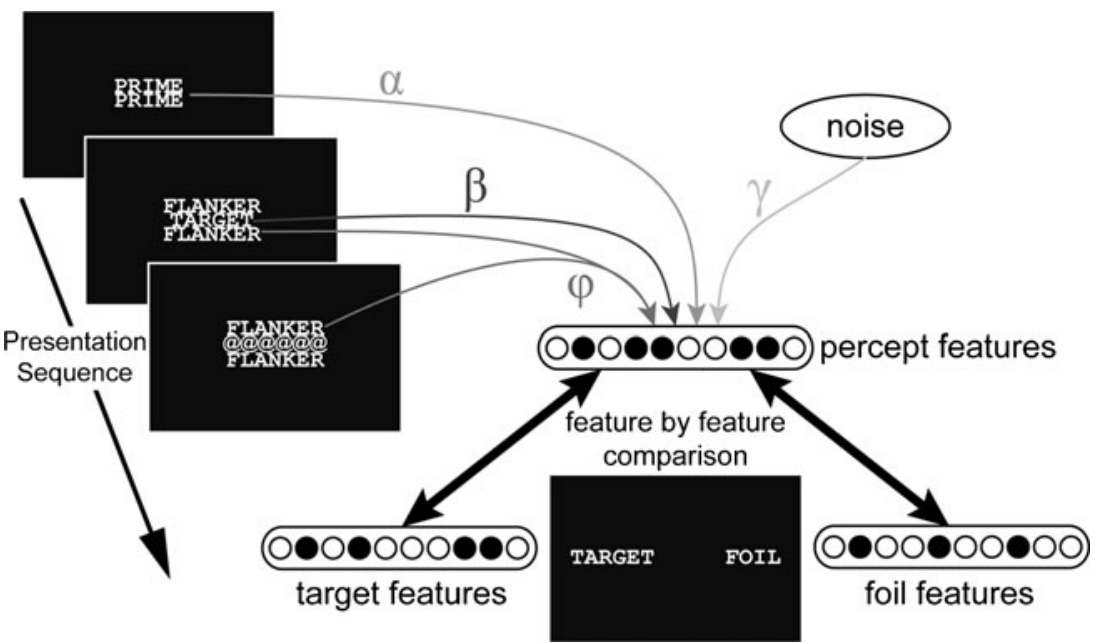

Fig. 4 In the responding-optimally-with-unknown-sources-of-evidence (ROUSE) model, words are composed of features that exist in an active or inactive state. Percept features can be independently be activated by four sources of evidence: the prime (with probability $\alpha$ ), the target (with probability $\beta$ ), the flanker (with probability $\varphi$ ) and visual noise (with probability $\gamma$ ). Since the source(s) of activation is unknown, these activation parameters indicate the probability that a feature remains active and indistinguishable from target activation, until the decision process is initiated. During the decision process, the current percept is compared with the features of the two choice options, the target and the foil 
probability $\beta$; and noise, with probability $\gamma$. The system is assumed to have access only to what features are active and not the source of their activation (i.e., source confusion). The probabilistic effect of $\alpha$, if not counteracted, will lead to a preference for whichever choice alternative is primed in the case of naive decision. Analogously, the probabilistic effect of $\varphi$, if not counteracted, will lead to a preference for whichever choice alternative is the same as a flanker.

These positive preferences are counteracted by a decision process that assigns lower levels of evidence to features that might have been activated by nontarget words that are simply nearby in time and space (i.e., discounting). ROUSEa posits that perceptual activation and inference is based on four independently driven sources of letter activation: the target, the prime, the flanker, and random visual noise. To discount features optimally, the system would need to know the "true" probabilities that each of these sources contributes the relevant feature to the percept (i.e., $\alpha, \varphi, \beta$, and $\gamma$ ). However, the decisional system does not have access to the exact probabilities, because the source of perceived letters is often unknown and therefore uses estimates of feature activation probabilities $\left(\alpha^{\prime}, \varphi^{\prime}, \beta^{\prime}\right.$, and $\left.\gamma^{\prime}\right)$ to infer the probability that a choice word is or is not the target according to the observed pattern of feature activation. Feature activation probability estimates are used to calculate the evidence provided by each feature.

Feature evidence takes the form of a likelihood ratio specifying the probability that a feature is from the target word over the probability that the feature is part of the foil, given the feature's current activation state, whether or not the feature appeared in the prime, and whether or not the feature appeared in the flanker. The feature likelihood ratios given all of these contingencies are presented in Fig. 5. The contingency table in Fig. 5 shows that the feature likelihood ratio assigned to an active feature that might have arisen from the prime (row 2, right column), the flanker (row 3, right column), or both (bottom right) is at a lower discounted level when compared with the ratio for the case when the feature could have only been activated by the target (top right). These discounted evidence levels serve to counteract source confusion by reducing the target evidence that active features would otherwise provide.

Assuming conditional independence between the features, feature evidence can be multiplied together across all word features, both active and inactive, to produce an overall likelihood that the choice word is the target. The odds of a target choice over a foil choice are given by the following ratio:

$\Phi\left(\frac{\mathrm{T}}{\mathrm{F}}\right)=\frac{\prod_{i=1}^{N} \frac{p\left(V\left\{\mathrm{~T}_{i}\right\} \mid \mathrm{T} \text { is target }\right)}{p\left(V\left\{\mathrm{~T}_{i}\right\} \mid \mathrm{T} \text { is foil }\right)}}{\prod_{j=1}^{N} \frac{p\left(V\left\{\mathrm{~F}_{i}\right\} \mid \mathrm{F} \text { is target }\right)}{p\left(\mathrm{~V}\left\{\mathrm{~F}_{i}\right\} \mid \mathrm{F} \text { is foil }\right)}}$

where $\mathrm{T}$ and $\mathrm{F}$ refer to the target and foil word, $\mathrm{N}$ is the total number of features, and $V\left(\mathrm{~T}_{i}\right)$ and $V\left(\mathrm{~F}_{i}\right)$ represent binary values denoting the $\mathrm{ON}$ and $\mathrm{OFF}$ states of activation of the
Feature DID

NOT Appear State Feature Activation

in Flanker

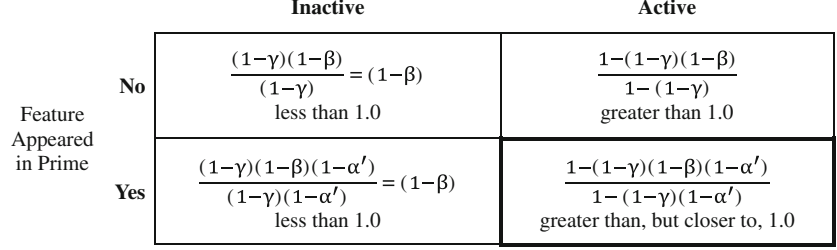

Feature DID

Appear in State Feature Activation

Flanker

Active

\begin{tabular}{|c|c|c|}
\hline Feature & $\begin{array}{c}\frac{(1-\gamma)(1-\beta)\left(1-\varphi^{\prime}\right)}{(1-\gamma)\left(1-\varphi^{\prime}\right)}=(1-\beta) \\
\text { less than } 1.0\end{array}$ & $\begin{array}{l}\frac{1-(1-\gamma)(1-\beta)\left(1-\varphi^{\prime}\right)}{1-(1-\gamma)\left(1-\varphi^{\prime}\right)} \\
\text { greater than, but closer to, } 1.0\end{array}$ \\
\hline Yes & $\frac{(1-\gamma)(1-\beta)\left(1-\alpha^{\prime}\right)\left(1-\varphi^{\prime}\right)}{(1-\gamma)\left(1-\alpha^{\prime}\right)\left(1-\varphi^{\prime}\right)}=(1-\beta)$ & $\begin{array}{l}\frac{1-(1-\gamma)(1-\beta)\left(1-\alpha^{\prime}\right)\left(1-\varphi^{\prime}\right)}{1-(1-\gamma)\left(1-\alpha^{\prime}\right)\left(1-\varphi^{\prime}\right)} \\
\text { greater than, but closest to, } 1.0\end{array}$ \\
\hline
\end{tabular}

Fig. 5 The evidence provided by each feature in the form of featurelikelihood ratios: the probability that a feature exists in its on/off state given that it is part of the target (and not the foil) over the probability of its state given that it is part of the foil (and not the target). Features that are shared between the target and foil do not provide discriminating information and are ignored in the decision process. This $2 \times 2 \times 2$ contingency table shows the evidence provided by a feature according to whether the feature is active or inactive, in the prime or not in the prime, and in the flanker or not in the flanker. As in Fig. $4, \alpha, \varphi, \beta$, and $\gamma$, respectively, refer to the probabilities of feature activation by the prime, flanker, target, and noise. In theory, estimates of these probabilities should be used. In practice, $\beta$, and $\gamma$ are assumed to be estimated correctly. The estimates of $\alpha$ and $\varphi$ (denoted $\alpha^{\prime}$ and $\varphi^{\prime}$ ) differ from their true values, which results in under- or overdiscounting of prime and flanker features

$i$-th feature of the target and the foil, respectively. The product in the numerator is across all feature likelihood ratios for the target word, which when combined gives the likelihood ratio of the target word. The product of the denominator is the likelihood ratio for the foil. Using this odds equation, the optimal response among words can be made. If the odds are greater than one, then the target choice is identified as the target, whereas if the odds are less than one, the foil is (incorrectly) identified as the target. In the case of ties (odds equal to 1), a random selection is made between the choice words.

Estimates of $\beta$ and $\gamma$ are theorized to be close to their true values. However, the true prime and flanker activation probabilities, $\alpha$ and $\varphi$, are believed to be harder to estimate accurately because they vary with the conditions of any visual setting. For instance, the actual and estimated intrusion rates are thought to vary with duration of the feature source. The model's behavior depends critically on the magnitude and direction of the difference between $\alpha$ and its estimate, $\alpha^{\prime}$, and the difference between $\varphi$ and its estimate $\varphi^{\prime}$. These differences are used by the system to set the respective levels of feature evidence discounting and to 
determine the amount and direction of priming and flanking effects. For example, the direction of the difference between $\alpha$ and $\alpha^{\prime}$ determines the direction of the priming effects: positive priming when $\alpha^{\prime}<\alpha$, and negative priming when $\alpha^{\prime}>\alpha$. Larger difference magnitudes correspond to larger priming effects.

The original ROUSE model included a parameter $\rho$ that represented the similarity between a prime and a given choice (i.e., the proportion of features in a target or foil choice option that match the prime and therefore could conceivably be activated by it). When the prime and choice are the same, $\rho$ is set to 1.0. When the prime and the choice are unrelated, $\rho$ is set to 0.0 . Although it is unrealistic to assume zero feature overlap, our efforts to ensure minimal letter overlap (one letter at most) led us to simplify by making this assumption. ROUSEa includes an analogous parameter, $\tau$, that represents the similarity between the flanker and a given choice. When the flanker and choice are the same, $\tau$ is set to 1.0 , indicating that all of the features in a (target or foil) choice option match the flanker and could be activated by it. As was done for prime similarity, we again simplify and assume $\tau$ is 0.0 when the flanker does not match a choice word.

\section{Applying the ROUSE model to the priming and flanking experiment}

The augmented ROUSE was fit to data by using an analytic form of the model (see Huber, 2006). In principle, the actual feature intrusion probabilities from primes and flankers should vary with their duration, but prior research has shown that the ROUSE predictions are determined almost entirely by the difference between the actual intrusion rate and the estimated intrusion rate. Thus, the actual intrusion rates for primes and flankers were set to single values that did not differ with prime or flanker duration, and only the estimated intrusion values were allowed to vary across conditions. Following the precedents set in earlier modeling, (e.g., Huber et al., 2001; Weidemann et al., 2005), it is assumed that estimates of feature activations resulting from targets and noise are equal to the actual values (i.e., $\beta^{\prime} \equiv \beta$ and $\left.\gamma^{\prime} \equiv \gamma\right)$. Furthermore, we assumed that feature activations resulting from the target $(\beta)$ vary with viewing conditions (see Huber et al., 2002b). Since both the primes and flankers acted as distractors in the present experiment, varying their duration may have made them more or less effective in masking target features. As such, for each of the four duration conditions, we included a separate $\beta$ parameter (however, for comparison, a nonduration dependent single $\beta$ model was briefly considered). The differential masking effects of both primes and flanker will be addressed in the discussion below.
Fitting the augmented ROUSE model involved estimating the following eleven parameters:

a. The probability that a choice word feature is activated by the prime $(\alpha)$,

b. The estimated probability that a feature is activated by a short duration prime $\left(\alpha_{S}^{\prime}\right)$,

c. The estimated probability that a feature is activated by a long prime $\left(\alpha_{L}^{\prime}\right)$,

d. The probability that a choice word feature is activated by the flanker $(\varphi)$,

e. The estimated probability that a feature is activated by a short duration flanker $\left(\varphi^{\prime} . S\right)$,

f. The estimated probability that a feature is activated by a long flanker $\left(\varphi^{\prime} .{ }_{L}\right)$,

g. The probability that a feature is activated due to the target flash when the prime and flanker are both short $\left(\beta_{S S}\right)$,

h. The probability that a feature is activated due to the target flash when the prime is short and flanker is long $\left(\beta_{S L}\right)$,

i. The probability that a feature is activated due to the target flash when the prime is long and flanker is short $\left(\beta_{L S}\right)$,

j. The probability that a feature is activated due to the target flash when the prime and flanker are both long $\left(\beta_{L L}\right)$,

k. The probability that a feature is activated due to noise $(\gamma)$.

The $\alpha^{\prime}, \varphi^{\prime}$, and $\beta$ parameters in the model vary as a function of duration condition. The subscripts indicate which parameters correspond to which duration conditions - the first indicating the prime duration and the second indicating the flanker duration. Although these parameters vary with duration condition, they do not vary with priming or flanking conditions.

The model was fit to 36 independent data points as depicted in Fig. 2. These data points are the averaged results of all participants. Each participant contributed at most 16 trials to each of the 36 conditions shown. The black dots overlaid on Fig. 2 show the augmented ROUSE models predictions. The independent model predictions from Fig. 2 are aggregated together to create the predictions seen in Fig. 3 (i.e., this figure does not show independent predictions, but instead dots are an average prediction from the appropriate conditions in Fig. 2). Model parameters were found by maximizing the likelihood of the aggregated responses of all participants. The best-fitting parameters for this model are: $\alpha=.145, \alpha_{S .}^{\prime}=.0793, \alpha_{L}^{\prime}=.214$, $\varphi=.124, \varphi^{\prime}{ }_{S}=.0778, \varphi^{\prime}{ }_{L}=.104, \beta_{S S}=.0621, \beta_{S L}=.0 .351$, $\beta_{L S}=.0980, \beta_{L L}=.0647$, and $\gamma=.0275$ (fit-value: $G^{2}=$ 179). ${ }^{1}$ The ratio of parameters (11) to data points (36) may seem a bit large, but many of the parameters were established

\footnotetext{
${ }^{1}$ In the present context, minimizing $G^{2}$ is the same as maximizing likelihood; that is, the best-fitting parameters presented are maximal likelihood estimates.
} 
in prior research, and the present values and their directions of variation match those found earlier. In addition, the model's parameters have clear interpretations. Given the relatively small number of trials per condition, fitting individual participants' data and averaging the resulting parameters are unlikely to outperform our group analysis approach in obtaining population parameter estimates (Cohen, Sanborn \& Shiffrin, 2008, have shown that as the amount of data per condition drops, fitting aggregated data becomes superior to fitting individual data prior to combining, since the amount of data per condition drops). As with any group analysis, there remains the possibility that some individuals are not well represented by the population average and therefore the model may not account for these individuals. We cannot assess this directly because of the limited data per condition, but individual differences may well have been reduced by the perceptual nature of the present study.

As can be seen in both Figs. 2 and 3, ROUSEa does a quite good job in accounting for the complex set of qualitative trends in the data. The model captures the robust priming effects: short primes producing positive priming and long primes producing negative priming (see Fig. 3A). The model produces these predictions by underdiscounting prime feature intrusions when the prime is short $\left(\alpha_{S .}^{\prime}<\alpha\right)$ and overdiscounting when the prime is long $\left(\alpha_{L}^{\prime}<\alpha\right)$. The model also captures the flanking effects presented in Fig. 3B. The flanker features are always underdiscounted, but the short flankers are discounted more (i.e., $\varphi^{\prime}{ }_{. S}<\varphi^{\prime}{ }_{L}<$ $\varphi)$. The larger positive flanking effect seen on the left half of Fig. 3B is reflected in this ordering. The features that intrude into the target percept from short flankers are not discounted as much as those from longer flankers. The discounting rate for long flankers is closer to actual intrusion rate and is therefore more optimal than for short flankers. ${ }^{2}$

ROUSEa not only accounts for the aggregated effects in Fig. 3, but also for many interesting interactions shown to a limited extent in Fig. 3 and more thoroughly in Fig. 2. Even though prime and flanker discounting vary solely as a function of duration, the patterns of observed interactions are captured well by the model. To fully capture the trends in the data, specifically the effect of duration conditions, it was necessary to assume that target perceptibility $(\beta)$ varied with prime and flanker duration. Prime duration influences on target perceptibility have been found in a number of previous priming studies and are straightforwardly accounted for by varying $\beta$, the target feature intrusion rate, across conditions \& Lyle, 2002). One way to justify this variation of $\beta$

\footnotetext{
$\overline{2}$ The difference between the magnitudes of $\varphi^{\prime}{ }_{. L}$ and $\varphi^{\prime}{ }_{S}$ although seemingly small $(.104-.078=.026)$, is hugely significant to the model fit. When the model is fit with these parameters equal, the fit value increases drastically to $G^{2}=398$. The difference in the model fits $\left(G_{\text {diff }}^{2}=219\right)$ is hugely significant, given the addition of a single parameter and the corresponding expected difference, $\chi^{2}(\alpha=.001, d f=1)=10.83$.
}

is through the differential masking effects of primes and flankers on target perception (which will be discussed shortly). The need to include such variation is seen clearly when ROUSEa is fit to the data assuming a single level of target perceptibility across all duration conditions (a single $\beta$ value is used). This restricted fit is presented in Figs. 2 and 3 as crosses. $^{3}$ This fit uniformly underpredicts accuracy in long prime, short flanker conditions (panel A2), and uniformly overpredicts accuracy in short prime, long flanker conditions (panel B1). These prediction errors can only be the result of differences in target perceptibility across duration conditions. This conclusion is most apparent when looking at the results from the NP/NF conditions (the first bar in each panel of Fig. 2). The four NP/NF conditions are control conditions that provide an indicator of baseline performance in each duration condition. In these conditions, the target and foil were unrelated to either the prime or flanker, so that the prime and the flanker do not contribute any features that would produce a preference toward either choice alternative. Accordingly, when the model generates NP/NF predictions, prime and flanker intrusion probabilities $(\alpha$ and $\varphi)$ and their estimates $\left(\alpha^{\prime}\right.$ and $\left.\varphi^{\prime}\right)$ do not enter into the calculations. Because of this, the restricted model necessarily makes the same prediction for the NP/NF condition regardless of prime or flanker duration; that is, the predicted accuracy is .79 in all four panels. Variations in target perceptibility $(\beta)$ across duration conditions naturally account for the observed differences in baseline performance that seem to depend solely on the duration of the primes and flankers, regardless of the features they provide. Nevertheless, it is noteworthy that the fit of the restricted model still exhibits most of the gross trends in the data by having prime and flanker discounting vary only as a function of duration, thus affirming the importance of differential discounting to ROUSEa's ability to account for the many interactions in the data.

\section{Discussion}

Our present experiment and modeling results provide evidence that, for perceptual identification of briefly flashed and masked targets, the mechanisms of source confusion and discounting operate for both primes and flankers. The model treats both primes and flankers as feature sources for the target percept, and these features are then discounted in not quite optimal fashion. More indirectly, the model treats both primes and flankers as sources of perceptual masking. These effects are seen in the model as variations of the $\beta$

\footnotetext{
${ }^{3}$ For completeness, the best-fit parameter values for this restricted ROUSE model are $\alpha=.149, \alpha_{S .}^{\prime}=.0789, \alpha_{L .}^{\prime}=.213, \varphi=.125, \varphi^{\prime} . S$ $=.0789, \varphi^{\prime}{ }_{L}=.108, \beta=.0652$, and $\gamma=.0322$ (fit-value: $G^{2}=1235$ ). These values are very similar to the previously reported parameters values, save the single $\beta$ value.
} 
parameter that determines the probability of feature extraction from the flashed target.

The primary findings of interest are the variations in the effect that both primes and flankers have as a function of their durations. Replicating earlier research, short primes produced positive priming (a tendency to choose the alternative matching the prime), and long primes produced negative priming (a tendency to choose the alternative that does not match the prime). In the model, this switch is the result of the system calculating evidence with an estimate of the actual prime feature intrusion rate $(\alpha=.145)$ that is too low for short primes $\left(\alpha_{S}^{\prime}=.0793\right)$ and too high for long primes $\left(\alpha_{L .}^{\prime}=.214\right)$.

Flanker effects were also dependent on the duration of the flanker and were explained in ROUSEa as a function of more discounting for longer flankers. However, discounting was generally reduced for flankers of all durations, so even the long flankers did not produce a negative effect. In particular, the flanker effects were captured in the model with an estimate of the feature intrusion rate from flankers that was higher for long $\left(\varphi^{\prime}{ }_{L}=.104\right)$ than for short flankers $\left(\varphi^{\prime}{ }_{S}=.0778\right)$, but both estimates were lower than the "actual" intrusion rate $(\varphi=.124)$. Given this trend, it is possible that an even longer, or otherwise more salient, flanker would produce overdiscounting. However, it is also conceivable that overdiscounting of flanker features is not possible. Discounting may depend on the perceived order of events in the visual system, and item presentation events may be marked preferentially by their onset, because after onset the system begins to accommodate to their presence. Thus, overdiscounting might be restricted to long primes with onsets well prior to the target flash. Whether overdiscounting, and "negative flanking" effects can be achieved must be left for future investigations.

According to the estimated target feature activation probabilities for each duration condition $\left(\beta_{S S}, \beta_{S L}, \beta_{L S}, \beta_{L L}\right)$, it appears that target perceptibility was the highest when the prime was long and the flanker was short, and the lowest when the reverse was the case: The prime was short and the flanker was long $\left(\beta_{S L}<\beta_{S S}<\beta_{L L}<\beta_{L S}\right)$. Given that target perceptibility generally dictates the likelihood that a target is correctly identified, it should be no surprise that this ordering coincides with participant performance across duration conditions (most easily seen when one focuses on the NP/ NF performance in Fig. 2). In general, a larger $\beta$ value uniformly increases performance across all priming and flanking conditions. Note that the only differences between the ROUSEa (dots) and restricted ROUSEa (crosses) predictions in Fig. 2 are the $\beta$ values, which visually just seem to slide the predictions of the nine within-duration conditions either up or down. The possibility that these effects are the result (in part) of masking will be discussed shortly. Here, we note that it would also be possible to produce similar patterns of predictions by varying $\gamma$ rather than $\beta$. The noise parameter, $\gamma$, also determines overall performance, because as noise increases, target identification performance drops. When fit to the data, a model with four $\gamma$ parameters (and one $\beta$ parameter) can similarly produce the established performance order (with $\gamma_{S L}>\gamma_{S S}>\gamma_{L L}>\gamma_{L S}$ ). The implication of this model is that different prime and flanker durations bring about different levels of noise features in the system. This differential noise model does a reasonable job of accounting for the trends in the data, but does not fit as well as the model with four $\beta$ parameters. ${ }^{4}$ Both versions provide a mechanism by which target perceptibility is the lowest when there are short primes and long flankers, and so on. We acknowledge that either process could account for the observed results and that both could be operating. Conceptually, it seems more natural to link the differential effects directly to the extraction of features from the flashed target, but future research would be needed to come to a more reliable conclusion.

\section{Masking effects}

That target perceptibility might vary with the duration of primes and flankers is a possibility consistent with the literature on masking effects, if one thinks of primes as forward masks and flankers as lateral and backward (metacontrast) masks. Huber (2008) has argued that shorter primes interfere more with accurate target flash perception because of their greater degree of forward masking, related to the possibility that long primes may have become habituated by the time the target appears (Huber \& O'Reilly, 2003). More generally, the onset of short primes is in close temporal proximity to the target onset, providing a narrow temporal window in which the inherent temporal "smearing" of the visual system will likely produce forward masking effects (see Breitmeyer, 2007, and Enns \& Di Lollo, 2000 , for useful reviews of masking).

Masking processes may similarly explain some of the effects caused by flankers. Differential masking by different duration flankers may be related to what is known as common-onset masking ( Di Lollo, Enns \& Rensink, 2000; Enns \& Di Lollo, 2000). In common-onset masking, a target and mask appear simultaneously. The mask appears spatially close to the target, but does not occlude any portion

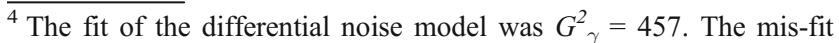
predominately occurs in the short prime, long flanker condition. To emulate the poor performance shown by participants in this condition, the estimated noise feature intrusion rate must be exceptionally high. However, at this high rate, noise begins to dampen the effects of prime features, which are actually the most extreme under these duration conditions.
} 
of it (i.e., the mask with its nonoverlapping contours is a metacontrast mask). When the offset of the mask is the same as the offset of the target, both stimuli are seen clearly and distinctly. However, if the mask remains on after the target offset, then target perceptibility is reduced. The prevailing explanation for why common-onset masking occurs is object substitution theory (Enns, 2004). The basis of this theory is that there is an active interplay between higher and lower levels of the visual system. When a target is removed, the sensory information at the lower level is no longer consistent with the higher level target hypotheses. If a mask remains occupying the space vacated by the target, then it will become the focus of visual processing, to the detriment of target identification. In the present experiment, short duration flankers had the same onset and offset as the target. As such, they would produce minimal masking of target features. However, the long duration flankers, which had the same onset, remained present after the target was removed. Thus, due to common-onset masking, it seems likely that long flankers mask target features more than short flankers do. In summary, the masking effects of both primes and flankers can reasonably be argued to operate together to produce the variations in performance that we captured in the model with variations of $\beta$ (and the same masking effects could be used to justify alternative variations of $\gamma$ ).

\section{Final note on relating priming effects and flanking effects}

In the present paradigm, we examined flanking and priming in a common paradigm and task requiring perceptual identification of a briefly flashed and masked target. The majority of explorations of flanking effects occur in quite different paradigms, ones in which flankers and targets are quite visible, have a limited number of identities known throughout testing, and in which RTs are the primary measure of interest. In these paradigms, it is quite possible that the flankers (as well as targets) receive automatic processing all the way to the output/response assignment stage, naturally producing congruency and response compatibility effects. Such mechanisms could well be operating in those paradigms but do not rule out the possible additional presence of the processes being explored in the present article (a question we leave for future research). Thus, the present research cannot be said to "bridge the gap" between the literatures on priming and flanking effects. Nonetheless, a first step has been taken in this direction by our results, and modeling of those results, that make a case for similar processes induced by the presence of primes and flankers upon the identification of target stimuli presented near identification threshold.
Author Note The present research was supported by National Science Foundation Grant 6804643 awarded to R.M.S.

\section{References}

Breitmeyer, B. G. (2007). Visual masking: Past accomplishments, present status, future developments. Advances in Cognitive Psychology, 3, 9-20.

Cohen, A. L., Sanborn, A. N., \& Shiffrin, R. M. (2008). Model evaluation using grouped and individual data. Psychonomic Bulletin \& Review, 15, 692-712.

Denton, S. E., \& Shiffrin, R. M. (in press). Short-term visual priming across eye movements. In C. Chubb, B. A. Dosher, Z.-L. Lu, \& R. M. Shiffrin (Eds.), Vision-Memory-Attention. Washington, DC: American Psychological Association.

Di Lollo, V., Enns, J. T., \& Rensink, R. A. (2000). Competition for consciousness among visual events: The psychophysics of reentrant visual processes. Journal of Experimental Psychology: General, 129, 481-507.

Enns, J. T. (2004). Object substitution and its relation to other forms of visual masking. Vision Research, 44, 1321-1331.

Enns, J. T., \& Di Lollo, V. (2000). What's new in visual masking? Trends in Cognitive Sciences, 4, 345-352.

Eriksen, B. A., \& Eriksen, C. W. (1974). Effects of noise letters upon the identification of a target letter in a nonsearch task. Perception \& Psychophysics, 16, 143-149.

Eriksen, C. W. (1995). The flanker task and response competition: A useful tool for investigating a variety of cognitive problems. Visual Cognition, 2, 101-118.

Gratton, G., Coles, M. G. H., \& Donchin, E. (1992). Optimizing the use of information: Strategic control of activation of responses. Journal of Experimental Psychology: General, 121, 480-506.

Gratton, G., Coles, M. G. H., Sirevaag, E. J., Eriksen, C. W., \& Donchin, E. (1988). Pre- and poststimulus activation of response channels: A psychophysiological analysis. Journal of Experimental Psychology: Human Perception and Performance, 14, 331-344.

Hochhaus, L., \& Johnston, J. C. (1996). Perceptual repetition blindness effects. Journal of Experimental Psychology: Human Perception and Performance, 22, 355-366.

Huber, D. E. (2006). Computer simulations of the ROUSE model: An analytic simulation technique and a comparison between the error variance-covariance and bootstrap methods for estimating parameter confidence. Behavior Research Methods, 38, 557-568.

Huber, D. E. (2008). Immediate priming and cognitive aftereffects. Journal of Experimental Psychology: General, 137, 324-347.

Huber, D. E., \& O'Reilly, R. C. (2003). Persistence and accommodation in short-term priming and other perceptual paradigms: Temporal segregation through synaptic depression. Cognitive Science, 27, 403-430.

Huber, D. E., Shiffrin, R. M., Lyle, K. B., \& Quach, R. (2002a). Mechanisms of source confusion and discounting in short-term priming 2: Effects of prime similarity and target duration. Journal of Experimental Psychology: Learning, Memory, and Cognition, 28, 1120-1136.

Huber, D. E., Shiffrin, R. M., Lyle, K. B., \& Ruys, K. I. (2001). Perception and preference in short-term word priming. Psychological Review, 108, 149-182.

Huber, D. E., Shiffrin, R. M., Quach, R., \& Lyle, K. B. (2002b). Mechanisms of source confusion and discounting in short-term priming: 1. Effects of prime duration and prime recognition. Memory \& Cognition, 30, 745-757.

Kinoshita, S., \& Norris, D. (2009). Transposed-letter priming of prelexical orthographic representations. Journal of Experimental Psychology: Learning, Memory, and Cognition, 35, 1-18. 
Kučera, H., \& Francis, W. N. (1967). Computational analysis of presentday American English. Providence, RI: Brown University Press.

Norris, D., \& Kinoshita, S. (2008). Perception as evidence accumulation and Bayesian inference: Insights from masked priming. Journal of Experimental Psychology: General, 137, 434-455.

Sanborn, A. N., Malmberg, K. J., \& Shiffrin, R. M. (2004). High-level effects of masking on perceptual identification. Vision Research, 44, 1427-1436.

Straw, A. D. (2008). Vision Egg: An open-source library for realtime visual stimulus generation. Frontiers in Neuroinformatics, 2, 1-10.
Weidemann, C. T., Huber, D. E., \& Shiffrin, R. M. (2005). Confusion and compensation in visual perception: Effects of spatiotemporal proximity and selective attention. Journal of Experimental Psychology: Human Perception and Performance, 31, 40-61.

Weidemann, C. T., Huber, D. E., \& Shiffrin, R. M. (2008). Prime diagnosticity in short-term repetition priming: Is primed evidence discounted, even when it reliably indicates the correct answer? Journal of Experimental Psychology: Learning, Memory, and Cognition, 34, 257-281. 